\title{
Effects of Lorentz Symmetry Violation in the Polarization of Gravitational Waves
}

\author{
Mapse Barroso F. Filho*, Kevin M. Amarilo and Roberto V. Maluf \\ Universidade Federal do Ceará, Brazil \\ E-mail: mapsedfisica.ufc.br, kevinefisica.ufc.br, \\ r.v.malufefisica.ufc.br
}

\begin{abstract}
This work aims to solve Einstein Equation in a scenario with Lorentz symmetry violation for gravitational waves polarization study. It will be commented about Standard Model Extension, spontaneous and explicit violation of a symmetry as well as their consequences. The bumblebee model will be used for the study of Lorentz violation, where the terms that breaks off the symmetry are included in the Lagrangean of the gravitational theory. The Euler-Lagrange equations are used for determination of modified graviton propagator, where we expend the Lagrangean up to second order of the perturbation field. We conclude that, in this scenario, the dispersion relation of graviton is different of the usual one, where we have a term that selects a preferred direction in the spacetime. Besides that, the graviton still with two degrees of freedom, despite the existence of bumblebee field. Then, the modified wave equation for perturbation field is solved and we compare the polarization states of the gravitational wave solution modified with the usual case. We show that for a bumblebee field being timelike or in the same direction of wave momentum we have no changes in the polarization tensor. But for a bumblebee field being in another diretion we have a modified polarization tensor for the graviton.
\end{abstract}

International Conference on Black Holes as Cosmic Batteries: UHECRs and Multimessenger Astronomy BHCB2018

12-15 September, 2018

Foz do Iguaçu, Brasil

${ }^{*}$ Speaker. 


\section{Introduction}

Detection of gravitational waves was one of the most important events for the high energy physics of XXI century. In September 11th, 2016 the laboratory VIRGO and LIGO announced that the first detection was made in September 14th, 2015, in an event called GW150914. The second detection was done in December 26th, 2015, this event is called GW151226. The detected waves of both events are due to coalescence of two black holes located at distances of approximately 410 Mpc and $440 \mathrm{Mpc}$.

Furthermore, the main problem of current physics is the quantization of gravity. The standard model extension (SME), that is an effective field theory that mixes the standard model (SM) and Lorentz violating effects, is a powerful theory that describes all fundamental interactions as well as all particles in nature. Therefore, theories with Lorentz Symmetry Breaking (LSB) are of great interest of the modern research.

The existence of a nonzero vacuum expectation value for a field triggers a spontaneous LSB. The simplest field theory that a vector that acquires nonzero expectation values are the bumblebee models $[1,2]$. In this theory the LSB is triggered by a vector field, whose minimum potential gives rise to the background field.

In this work, we solve Einstein equations in a scenario with LSB for the gravitational waves polarization study. We start investigating the consequences of the spontaneous breaking of Lorentz symmetry, triggered by the bumblebee vector field. The action of the bumblebee term is varied and we determine the modified graviton propagator. Then, the modified wave equation for the perturbation field is solved and we compare the polarization tensor of the modified gravitational wave solution with the usual case.

\section{Bumblebee modifications in graviton modes}

The Lorentz Violation can be included in gravitational sector through the following action

$$
S=S_{E H}+S_{L V}+S_{M}
$$

where $S_{E H}$ is the Einstein-Hilbert (EH) action, given by

$$
S_{E H}=\int d^{4} x \sqrt{-g} \frac{2}{\kappa^{2}}(R-2 \Lambda)
$$

where $R$ is the Ricci curvature scalar and $\Lambda$ is the cosmological constant. Here, we will disregard the cosmological constant.

The action that accounts for the Lorentz violating terms is,

$$
S_{L V}=\int d^{4} x \sqrt{-g} \frac{2}{\kappa^{2}}\left(u R+s^{\mu v} R_{\mu v}+t^{\mu v \alpha \beta} R_{\mu v \alpha \beta}\right) .
$$

In this gravity model, when $B^{\mu}$ acquires a nonzero vacuum expectation value, it induces both Lorentz and diffeomorphism violations. The bumblebee model can be represented in this form,

$$
S_{B}=\int d^{4} x \sqrt{-g}\left[-\frac{1}{4} B_{\mu v} B^{\mu v}+\frac{2 \xi}{\kappa^{2}} B^{\mu} B^{v} R_{\mu v}-\frac{\kappa}{2}\left(B^{\mu} B_{\mu} \pm b^{2}\right)^{2}-B^{\mu} J_{\mu}\right] .
$$


For $t^{\mu v \alpha \beta}=0$ and,

$$
u=\frac{1}{4} \xi B^{\alpha} B_{\alpha}, \quad s^{\mu v}=\xi\left(B^{\mu} B^{v}-\frac{1}{4} g^{\mu v} B^{\alpha} B_{\alpha}\right),
$$

where the field strength is $B_{\mu v}=\partial_{\mu} B_{v}-\partial_{v} B_{\mu}$. In this case, $J^{\mu}$ is a conserved current composed of matter fields and it is also the source of $B_{\mu}$. It will be disregarded because we don't need the dynamics of the matter fields. The potential that triggers the LSB is,

$$
V=\frac{\kappa}{2}\left(B^{\mu} B_{\mu} \pm b^{2}\right)^{2}
$$

So, the symmetry violation happens whenever the constant $b^{2}$ is assumed in the potential. To determine the modified graviton propagator we need to linearize the Lagrangean. So, we can split the dynamic fields considering the vacuum expectation values and the quantum fluctuations

$$
\begin{gathered}
g_{\mu v}=\eta_{\mu v}+\kappa h_{\mu v}, \\
B_{\mu}=b_{\mu}+\tilde{B}_{\mu},
\end{gathered}
$$

where $h_{\mu \nu}$ and $\tilde{B}_{\mu}$ are small perturbations. Varying the action and using the method of Green's function, we can determine the solution for the linearized bumblebee equation in the momentum space. It is worth to mention that the Ricci tensor and the Ricci escalar are up to second order in $h_{\mu \nu}$. Therefore, following the steps according to [3], we have the Lagrangean

$$
\begin{aligned}
\mathscr{L}_{L V} & =\xi\left[p^{2} b_{\mu} b_{v} h^{\mu v} h_{\alpha}^{\alpha}+\frac{1}{2}\left(b_{\mu} p^{\mu}\right)^{2}\left(h_{\alpha}^{\alpha}\right)^{2}-\frac{1}{2}\left(b_{\mu} p^{\mu}\right)^{2} h^{\mu v} h_{\mu v}\right. \\
& \left.+p^{2} b_{\mu} b_{v} h^{\mu \alpha} h_{\alpha}^{v}-\left(b_{\mu} b_{v} p_{\alpha} p_{\beta}+b_{(\mu} p_{v)} b_{(\alpha} p_{\beta}\right) h^{\mu v} h^{\alpha \beta}\right] \\
& +\frac{4 \xi^{2}}{\kappa^{2}}\left[\left(-2 p^{2} b_{\mu} b_{v}-2 b^{2} p_{\mu} p_{v}+4 b_{\mu} p^{\mu} b_{(\alpha} p_{\beta)}-\frac{p^{2} p_{\mu} p_{v}}{4 \lambda}\right) h^{\mu v} h_{\alpha}^{\alpha}\right. \\
& +\left(2 b_{\mu} b_{v} p_{\alpha} p_{\beta}-b_{(\mu} p_{v)} b_{(\alpha} p_{\beta)}+\frac{b^{2} p_{\mu} p_{v} p_{\alpha} p_{\beta}}{p^{2}}-\frac{2 b_{\mu} p^{\mu} p_{\mu} p_{v} b_{(\alpha} p_{\beta)}}{p^{2}}\right. \\
& \left.+\frac{p_{\mu} p_{v} p_{\alpha} p_{\beta}}{4 \lambda}\right) h^{\mu v} h^{\alpha \beta}+\left(b^{2} p^{2}-\left(b_{\mu} p^{\mu}\right)^{2}+\frac{p^{4}}{4 \lambda}\right)\left(h_{\alpha}^{\alpha}\right)^{2} \\
& \left.+\left(p^{2} b_{\mu} b_{v}-2 b_{\mu} p^{\mu} b_{(\mu} p_{v)}+\frac{\left(b_{\mu} p^{\mu}\right)^{2} p_{\mu} p_{v}}{p^{2}}\right) h^{\mu \lambda} h_{\lambda}^{v}\right]+\mathscr{O}\left(h^{3}\right),
\end{aligned}
$$

where $p^{\mu}=\left(p_{0}, \mathbf{p}\right), \sigma=\frac{2 \xi}{\kappa^{2}}$. Hence, for analyzing the effects of the bumblebee field we need the kinetic Lagrangean,

$$
\mathscr{L}_{K I N}=\mathscr{L}_{E H}+\mathscr{L}_{L V}
$$

The graviton propagator is defined as

$$
\left\langle 0\left|T\left[h_{\mu v}(x) h_{\alpha \beta}(y)\right]\right| 0\right\rangle=D_{\mu v, \alpha \beta}(x-y),
$$

where the operator $D_{\mu v, \alpha \beta}(x-y)$ satisfies the Green's equation,

$$
\hat{\mathscr{O}}_{\lambda \sigma}^{\mu v} D^{\lambda \sigma, \alpha \beta}(x-y)=i \mathscr{I}^{\mu v, \alpha \beta} \delta^{(4)}(x-y) .
$$


Thus, the Feynman propagator is given by inverting $\hat{\mathscr{O}}_{\lambda \sigma}^{\mu v}$,

$$
D_{\lambda \sigma, \alpha \beta}(x-y)=i \hat{\mathscr{O}}_{\mu \nu \lambda \sigma}^{-1} \delta^{(4)}(x-y) .
$$

The whole process to find the Feynman propagator for $\mathscr{L}_{K I N}$ is out of the scope of this work. This can be made through the Barnes-Rivers projectors. Reference [3] show the whole process. In this work, they found two poles in graviton propagator. But, only one is physical and we will use it to our analysis. This pole is,

$$
\boxplus(p)=p^{2}+\xi(b \cdot p)^{2} .
$$

So, the equation of motion is given by,

$$
\hat{\mathscr{O}}_{\mu \nu \lambda \sigma} h^{\alpha \beta}=0 .
$$

By saturing this equation with respect to $p^{\mu} p^{v}, b^{(\mu} p^{v)}, b^{\mu} b^{v}$ and $\eta_{\mu v}$ we obtain the following constraints,

$$
\begin{gathered}
b_{\mu} b_{v} h^{\mu v}=0, \\
b_{(u} p_{v)} h^{\mu v}=0, \\
p_{\mu} p_{v} h^{\mu v}=0, \\
b_{\mu} h^{\mu v}=0, \\
p_{\mu} h^{\mu v}=0 .
\end{gathered}
$$

Besides that,

$$
h=0 .
$$

This set of equations gives to us a set of 12 constraints. So we can reduce the initial 14 degrees of freedom contained in the graviton and bumblebee fields. Consequently, there are just 2 degrees of freedom like the usual graviton. Hence, the modified equation of graviton is,

$$
\left[p^{2}+\xi(b \cdot p)^{2}\right] h_{\mu v}=0 .
$$

\section{Solutions of the modified graviton equation}

From Equation (2.22) we get the following dispersion relation,

$$
p_{0}=|\vec{p}|\left[\frac{\xi b^{0}|\vec{b}| \cos \Psi \pm \sqrt{1+\xi\left(b^{0}\right)^{2}-\xi|\vec{b}|^{2} \cos ^{2} \Psi}}{1+\xi\left(b^{0}\right)^{2}}\right]
$$

The most general solution for Equation (2.22) is

$$
h_{\mu v}(x)=\int \tilde{d}^{3} p \sum_{\lambda=1}^{4}\left[\varepsilon_{\mu \nu}^{(\lambda)} a^{(\lambda)}(p) e^{-i p \cdot x}+\varepsilon_{\mu \nu}^{*(\lambda)} a^{\dagger(\lambda)}(p) e^{i p \cdot x}\right]
$$

where, $\tilde{d}^{3} p=\frac{d^{3} p}{(2 \pi)^{3}\left|\sqrt{1+\xi\left(b^{0}\right)^{2}-\xi|\vec{b}|^{2} \cos ^{2} \theta}\right||2 \vec{p}|}$. 
Now, using Equations (2.19), (2.20) and (2.21) and choosing the direction of propagation in $z$ axis, the four momentum is $p^{\mu}=\left(p_{0}, 0,0, p_{3}\right)$. Therefore, we can see the modifications on the polarization tensor. We will consider some special cases. First, consider $b_{\mu}=\left(b_{0}, 0,0,0\right)$ or $b_{\mu}=\left(b_{0}, 0,0, b_{3}\right)$. In this case the, polarization tensor is,

$$
\varepsilon^{\mu v}=\left[\begin{array}{cccc}
0 & 0 & 0 & 0 \\
0 & \varepsilon^{11} & \varepsilon^{12} & 0 \\
0 & \varepsilon^{12} & -\varepsilon^{11} & 0 \\
0 & 0 & 0 & 0
\end{array}\right] .
$$

We see that this case is equal to the usual. We expected this, because in the first case, it was considered just a $b^{\mu}$ timelike and in the second one, no preferred direction was selected. However, if we consider the case where $b_{\mu}=\left(0,0, b_{2}, 0\right)$, we have,

$$
\varepsilon^{\mu \nu}=\left[\begin{array}{cccc}
e^{00} & e^{10} & 0-\frac{e^{00} p_{0}}{p_{3}} \\
e^{10} & -e^{00}\left(-1+\frac{p_{0}^{2}}{p_{3}^{2}}\right) & 0 & -\frac{e^{10} p_{0}}{p_{3}} \\
0 & 0 & 0 & 0 \\
-\frac{e^{00} p_{0}}{p_{3}} & -\frac{e^{10} p_{0}}{p_{3}} & 0 & \frac{e^{00} p_{0}^{2}}{p_{3}^{2}}
\end{array}\right] .
$$

Hence, the existence of a vacuum expectation value of the bumblebee field yields to a LSB. We see that the polarization tensor components do have dependence on components of the bumblebee field. So, the polarization tensor have dependence on the direction of this vector field.

\section{Conclusion}

In this work, it was investigated the effects of a Lorentz symmetry breaking due to an existence of a vector field. We used the simplest model in the literature, the bumblebee field. Here, we expand the Lagrangean of the theory up to the second order of $h_{\mu v}$ and we find the modified graviton propagator. With this, we get the modified graviton equation with a new relation of dispersion. Besides that, we get new constraints. Therefore, the new polarization tensor shows dependence on the bumblebee field.

\section{References}

[1] R. Bluhm and V. A. Kostelecký, Phys. Rev. D 71, 065008 (2005).

[2] R. Bluhm, S-H. Fung, and V. A. Kostelecký, Phys. Rev. D 77, 065020 (2008).

[3] MALUF, R. V. et al. Physical Review D, v. 90, n. 2, p. 025007, (2014).

[4] R. Bluhm, N. L. Gagne, R. Potting and A. Vrublevskis, Phys. Rev. D 77, 125007 (2008).

[5] Sean M. Carroll, Timothy R. Dulaney, Moira I. Gresham, and Heywood Tamx, Phys. Rev. D 79, 065011 (2009). 